\title{
EVIDENCE, EFFECTIVENESS AND EFFICIENCY IN BREAST CANCER RESEARCH
}

\author{
Rhana Pike and Bridie Carr \\ NHMRC Clinical Trials Centre \\ University of Sydney
}

This article describes the Cochrane Breast Cancer Group which was established in 1996 as an international, autonomous entity within the Cochrane Collaboration. The activities of the group are coordinated by its secretariat at the National Health and Medical Research Council Clinical Trials Centre in Sydney.

The main work of the Cochrane Collaboration is to conduct systematic reviews of the best available evidence for specific research questions. The information gathered allows clinicians and policy makers to identify the most effective and efficient treatments and allows investigators and funding bodies to refine plans for new research and avoid duplication.

The breast cancer group covers all aspects of the prevention, detection and treatment of breast cancer. It has two broad areas of operation:

- conducting systematic reviews for clinically relevant research questions

- maintaining and developing a specialised register of breast cancer trials as a resource for investigators.

\section{SYSTEMATIC REVIEWS}

When a question is suggested (either by a member of the group or by a health care professional or consumer), the secretariat checks that it has not previously been addressed. The question is then registered and a research protocol developed before the review commences. The protocol is then peer reviewed by individuals with methodologic or content expertise, and a consumer representative. The protocol, once accepted, is published on the Cochrane Database of Systematic Reviews as part of the Cochrane Library, so that other researchers are aware of it. A typical protocol topic, currently being investigated, is 'Effectiveness of different strategies for inviting women to participate in breast cancer screening'.

The systematic review is then conducted in accordance with the review protocol. It is generally accepted that a systematic review can take up to one year to complete. During this time, the editorial committee provides advice or guidance to reviewers, if required. The secretariat provides the reviewers with a list of eligible clinical trials from its specialised register.

\section{THE SPECIALISED REGISTER OF RANDOMISED CLINICAL TRIALS IN BREAST CANCER}

The breast cancer group has the ongoing task of finding, evaluating and classifying valid clinical trials in breast cancer. A comprehensive search strategy has been developed and is used to find publications indexed on international medical and scientific literature databases (such as Medline). To find articles that have not been indexed, trained hand searchers scrutinise journals, reports, and conference proceedings for items of value. Articles found are checked for relevance, classified and coded. The register is kept up to date with routine monthly searches of the various databases.

\section{HOW CANYOU CONTRIBUTE?}

Health professionals and consumers can contribute to the work of the breast cancer group in various ways by:

- being part of a team conducting a systematic review

- articulating a new review question or developing a protocol

- evaluating the content and design of protocols for systematic reviews

- notifying the secretariat of unpublished research in breast cancer (such as conference presentations of trial designs or results)

- hand searching journals and meeting proceedings to identify randomised controlled trials

- searching for and translating material in languages other than English

- identifying sources of support

- disseminating the results of completed systematic reviews. Fit

The Cochrane Collaboration aims to help people make well-informed decisions about health care by preparing, maintaining and promoting the accessibility of systematic reviews of the effects of health care interventions. For further information contact Davina Ghersi, Review Group Coordinator (email: davina@ctc.usyd.edu.au); or Bridie Carr, Assistant Review Group Coordinator (email: bridie@ctc.usyd.edu.au); or write to the Cochrane Breast Cancer Group, NHMRC Clinical Trials Centre, Locked Bag 77, Camperdown NSW 1450; phone: (02) 9562 5000; or fax: (02) 95651863. 